\title{
Heuristic Rating Estimation Approach to The Pairwise Comparisons Method
}

\author{
Konrad Kułakowski \\ Department of Applied Computer Science, \\ AGH University of Science and Technology \\ Al. Mickiewicza 30, \\ 30-059 Cracow, Poland \\ konrad.kulakowski@agh.edu.pl
}

\begin{abstract}
The Heuristic Ratio Estimation (HRE) approach proposes a new way of using the pairwise comparisons matrix. It allows the assumption that the weights of some alternatives (herein referred to as concepts) are known and fixed, hence the weight vector needs to be estimated only for the other unknown values. The main purpose of this paper is to extend the previously proposed iterative $H R E$ algorithm and present all the heuristics that create a generalized approach. Theoretical considerations are accompanied by a few numerical examples demonstrating how the selected heuristics can be used in practice.
\end{abstract}

\section{Introduction}

The first evidence of the usage of pairwise comparisons (herein abbreviated as PC) comes from Ramon Llull (the XIII century) [935], then the method was rediscovered in the XIX century by Fechner [12]. In the first half of the twentieth century it was developed by Thurstone [38]. The Analytic Hierarchy Process $(A H P)$, introduced by Saaty [33], was another important extension to the PC theory, providing handy methods for dealing with the large number of criteria. Many examples demonstrate the usefulness of the method [3916 26 37]. Despite its long existence, research in the field of the PC research is still conducted. This is evidenced by the works discussing the strengths and weaknesses of the most popular $A H P$ approach [1131/3 34 2, and also by the works proposing the new $P C$ paradigms, and exploring the new areas of applicability, such as the Rough Set theory approach [15], fuzzy $P C$ relation handling [27|1341442, incomplete $P C$ relation [6]14]22], data inconsistency reduction [24], non-numerical rankings [20] and others. A broader discussion of the $P C$ method can be found in 3617 .

The newly proposed HRE approach [25] explores the use of the $P C$ method in cases when some alternatives (herein referred to as concepts) have known and fixed priorities. Therefore, it divides the concepts into two sets - initially known elements $C_{K}$ for which the weights are fixed and unknown elements $C_{U}$ for which the weights need to be determined. Then, by iteratively averaging the available weights (initially only the weights of elements from $C_{K}$ are available), subsequent propositions of the weight vector are computed.

The notion inherently integrated with the $P C$ method is data inconsistency [87]. If the data are fully consistent then any single comparison provides enough information about the relative order and the intensity of preferences of two concepts. In such a case, after performing $n-1$ comparisons, the weights of all $n$ concepts can be easily determined, provided that the $\mathrm{n}-1$ comparisons involve all the concepts. Thus, any special way of data processing in order to derive the priorities of concepts is not needed. If the input data are inconsistent the best thing to be done is to propose a heuristic that, despite the data inconsistency, allows the weights of concepts to be calculated.

The presented work is a follow-up of [25]. It introduces the new ideas (the lack of reciprocity or the lack of data) and presents the concepts introduced in $[25$ in a more systematic and formal way. The HRE approach presented in this article includes four complementary heuristics that are useful for calculating weights when the reference set of initially known elements $C_{K}$ is given (Sec. 3). Besides theoretical consideration the article examines the $H R E$ weight derivation procedures on a few numerical examples (Sec. 4). The article is opened by two sections introducing the $P C$ method (Sec. 1 and 21). A brief summary is provided in (Sec. (5). Additional explanations and definitions are placed in the appendices. 


\section{A pairwise comparisons method}

Man always has to make choices. Therefore he/she always has to make comparisons. The best bet is when one (the better one) needs to be selected from a pair. People are accustomed to this type of comparison. In daily contact, in the market, where paying for a fruit everyone is trying to choose the heavier one. The relative weight of two fruits that look like they are the greatest can be easily estimated by comparing the weight of the fruit held in one hand with the weight of the fruit held in the other hand. Usually, making the right choice is possible without any additional tools indicating weight. In reality people have to compare much more complicated things than fruits. Often there is no way to make an accurate comparison. There is no 'weight' for the problem. Even worse, usually there are many different things that need to be compared. In such a case the PC approach comes to the rescue. It allows people to do what they do best - comparing pairs. The final synthesis of partial assessments is performed in accordance with predefined algorithms, such as the eigenvalue method or geometric mean method [19].

The input data to the $P C$ method is a $(P C)$ matrix $M=\left(m_{i j}\right)$ and $m_{i, j} \in \mathbb{R}_{+}$where $i, j \in\{1, \ldots, n\}$ represents partial assessments over the finite set of concepts $C \stackrel{d f}{=}\left\{c_{i} \in \mathscr{C}, i \in\{1, \ldots, n\}\right\}$ where $\mathscr{C} \neq \emptyset$ is a universe of concepts. Let $\mu: C \nrightarrow \mathbb{R}_{+}$be a partial function that assigns to some concepts from $C \subset \mathscr{C}$ positive values from $\mathbb{R}_{+}$. Thus, the value $\mu(c)$ represents the importance of $c$. The output of the $P C$ method is the function $\mu$ defined for all $c \in C$. It introduces the total order in $C$ and usually will be written in the form of a vector of weights $\mu \stackrel{d f}{=}\left[\mu\left(c_{1}\right), \ldots \mu\left(c_{n}\right)\right]^{T}$ (see Fig. 1).

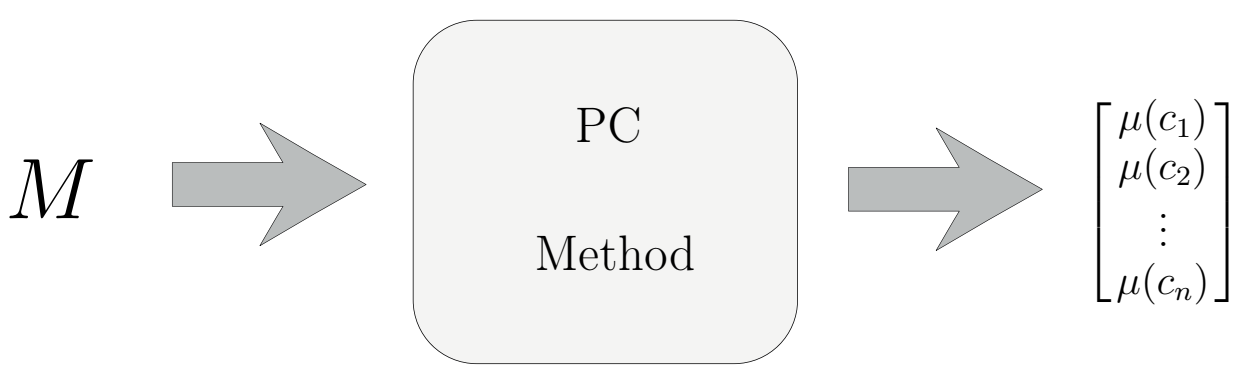

Fig. 1. PC Method input-output scheme

Concepts, originally referred to in the literature as subjective stimuli [38, alternatives [2] or activities [30], represent objects for which the relative importance indicators $m_{i j}$ and $m_{j i}$ need to be assessed.

It is assumed that, according to the best knowledge of experts, the importance of $c_{i}$ equals $m_{i j}$ of the importance of $c_{j}$ i.e. $\mu\left(c_{i}\right)=m_{i j} \mu\left(c_{j}\right)$. The matrix $M$ is said to be reciprocal if $\forall i, j \in\{1, \ldots, n\}: m_{i j}=\frac{1}{m_{j i}}$. This property reflects the intuition that if the relative importance ratio $c_{i}$ to $c_{j}$ is $m_{i j}$ then the the importance ratio $c_{j}$ to $c_{i}$ should be $1 / m_{i j}=m_{j i}$. However, intuitive reciprocity may not always be met. The matrix $M$ without reciprocity property is sometimes referred to in the literature as a generalized $P C$ matrix [23].

Ideally $M$ is also consistent i.e. $\forall i, j, k \in\{1, \ldots, n\}: m_{i j} \cdot m_{j k} \cdot m_{k i}=1$. Unfortunately, the knowledge stored in the $P C$ matrix usually comes from different experts, the consistency condition may not be met. In such a case, reasoning using $M$ may give ambiguous results. This leads to the data consistency (and inconsistency) concept formalized in the form of the inconsistency index. There are several different inconsistency indexes, including the Eigenvector Method [33, Least Squares Method, Chi Squares Method [7, Koczkodaj's distance based inconsistency index [21] and others. The most popular eigenvalue based approach [33] defines the consistency index (sometimes referred as the consistency ratio) as

$$
C I=\frac{\lambda_{\max }-n}{n-1}
$$

where $\lambda_{\max }$ is the principal eigenvalue of $n \times n$ matrix $M$. The iterative $H R E$ algorithm 25] adopts the last of them as a convenient and easy to use 'gauge' of data inconsistency. Koczkodaj's inconsistency index $\mathscr{K}$ of $n \times n$ and $(n>2)$ reciprocal matrix $M$ is equal to: 


$$
\mathscr{K}(M)=\max _{i, j, k \in\{1, \ldots, n\}}\left\{\min \left\{\left|1-\frac{m_{i j}}{m_{i k} m_{k j}}\right|,\left|1-\frac{m_{i k} m_{k j}}{m_{i j}}\right|\right\}\right\}
$$

where $i, j, k=1, \ldots, n$ and $i \neq j \wedge j \neq k \wedge i \neq k$.

There are also several different methods of deriving the weights vector out of the matrix $M$ [34]19]. Two the most popular are the eigenvector method [33] and the geometric mean method. According to the first one, the output $\mu$ (denoted as $\mu_{\mathrm{EV}}$ ) is the rescaled principal eigenvector of $M$, i.e.:

$$
\mu_{E V}=\left[\frac{v_{1}}{s_{E V}}, \ldots, \frac{v_{n}}{s_{E V}}\right]^{T} \quad \text { where } s_{E V}=\sum_{i=1}^{n} v_{i}
$$

and $v=\left[v_{1}, \ldots, v_{n}\right]^{T}$ is the principal eigenvector of $M$. The second method [10] proposes the adoption of rescaled geometric means of rows of $M$ as the output $\mu$. Thus,

$$
\mu_{G M}=\left[\frac{g_{1}}{s_{G M}}, \ldots, \frac{g_{n}}{s_{G M}}\right]
$$

where

$$
g_{i}=\left(\prod_{j=1}^{n} m_{i j}\right)^{1 / n} \quad \text { and } \quad s_{G M}=\sum_{i=1}^{n} g_{i}
$$

Other the priority deriving methods in the $A H P$ approach can be found in $[19] 18] 40]$.

\section{The HRE Algorithm Approach}

The HRE approach to the rating estimation in the pairwise comparisons method is based on a few intuitive heuristics. The first of them concerns dividing the set of concepts into known (reference) and unknown elements. Initially, $\mu$ is defined only for reference elements. Hence, only these elements can be used to estimate $\mu$ for unknown elements. With every subsequent step $\mu$ is specified for more and more elements. Thus, increasing the number of elements could be taken into account during calculations. The weights of initially known reference elements remain unchanged. Thus, the subsequent updates affect only unknown elements. In every step weights for unknown elements are determined as the arithmetic mean of determined values and the appropriate ratios (6). This iterative procedure forms an averaging with respect to the reference heuristics (a more detailed description in Sec. 3.1). Therefore, comparing with the eigenvalue based method, the HRE approach requires additional information about the reference elements (see Fig. 2).

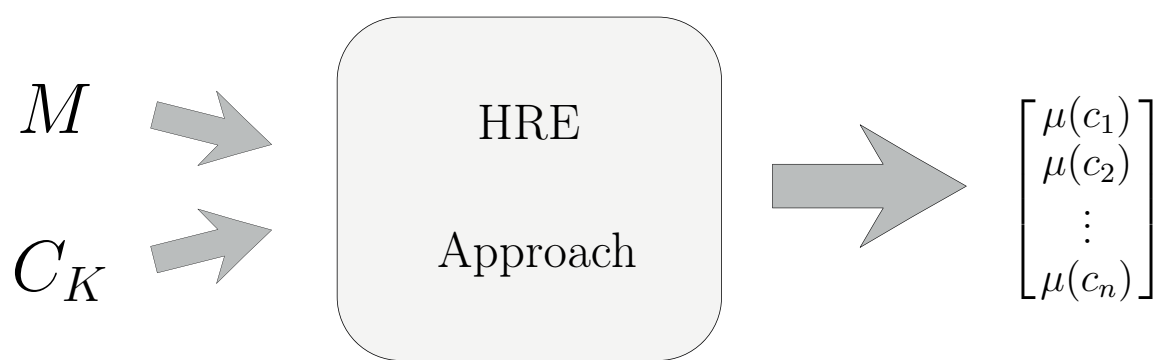

Fig. 2. $H R E$ approach input-output scheme. $C_{K}$ means the non-empty set of reference concepts.

In fact, sometimes the HRE procedure is equivalent to finding a solution for some linear equation system. Hence, if this equation system has an admissible solution, then its solution can be adopted as the output of 
the $H R E$ algorithm. If not, the weight vector needs to be determined with the help of the second heuristic (Sec. 3.2) as explained later in the work.

In general, the pairwise comparisons method assumes that the input matrix is reciprocal. It means that the ratio $m_{i j}$ expressing the relative importance of $c_{i}$ compared to $c_{j}$ should be the inverse of $m_{j i}$. Unfortunately, this assumption may not always be met [24]. Since the situation when $m_{i j} \neq 1 / m_{j i}$ is undesired, but possible in practice, the third heuristic proposes a simple method for calculating the new values $\widehat{m}_{i j}, \widehat{m}_{j i}$ so that they are mutually reciprocal and possibly close to the original $m_{i j}, m_{j i}$. The operation is called reciprocity restoration and is applied to any matrix $M$, which is processed by the $H R E$ algorithm.

The fourth heuristic addresses the problem of incomplete data, where not all the ratios $m_{i j}$ are known. Some of them can be recovered based on the assumed reciprocity. However, if both $m_{i j}$ and its counterpart $m_{j i}$ are unknown the reciprocity property does not help. In such a case, either the missing ratios are reconstructed [622] so that the standard methods can deal with the reconstructed matrix, or the procedure alone has to deal with the problem of missing matrix entries. The iterative $H R E$ approach does not need the matrix reconstruction. During the course of the iterative procedure, the new ratio values are computed using only those defined concepts that are reachable due to the availability of an appropriate ratio. In other words, if some ratio is missing all the multiplications which use the missing ratio are excluded from the basic update formula (6).

\subsection{Heuristics of averaging with respect to reference values}

The iterative averaging approach presented in [25] assumes that the set of concepts $C=C_{K} \cup C_{U}$ and $C_{K} \cap C_{U}=\emptyset$, where $C_{K}$ denotes concepts for which the actual value $\mu$ is initially known, and $C_{U}$ contains concepts for which the value $\mu$ needs to be determined. The relation between different concepts in $C$ is represented by $M$ so that in the case of the fully consistent matrix it holds that $\mu\left(c_{i}\right) m_{j i}=\mu\left(c_{j}\right)$. Hence, for a known, complete matrix $M$ and $c_{i} \in C_{K}, c_{j} \in C_{U}$ determining $c_{j}$ boils down to the performance of a single multiplication. Since $M$ is usually inconsistent, the $H R E$ algorithm considers $m_{j i} \mu\left(c_{i}\right)$ as a sample of $\mu\left(c_{j}\right)$, where the expected value of $\mu\left(c_{j}\right)$ is the arithmetic mean of the values $m_{j i} \mu\left(c_{i}\right)$. Of course, not all the values $\mu\left(c_{i}\right)$ are defined at the very beginning, but only those for which $c_{i} \in C_{K}$. Hence, in the first step of the $H R E$ procedure, the values $\mu\left(c_{j}\right)$ are estimated only on the basis of the initially known concepts. However, in the second step (assuming that $M$ is complete) all the other values $\mu\left(c_{i}\right)$ computed during the first step (for $i \neq j$ and $c_{i} \in C_{K} \cup C_{U}$ ) can be used to determine $\mu\left(c_{j}\right)$. Thus, for every concept $c_{j} \in C_{K}$ the r'th subsequent estimation of $\mu_{r}\left(c_{j}\right)$ computed by the $H R E$ iterative procedure (see [25]) meets the equation:

$$
\mu_{r}\left(c_{j}\right)=\frac{1}{\left|C_{j}^{r-1}\right|} \sum_{c_{i} \in C_{j}^{r-1}} m_{j i} \mu_{r-1}\left(c_{i}\right)
$$

where

$$
C_{j}^{r-1}=\left\{c \in C: \mu_{r-1}(c) \text { is known and } c \neq c_{j}\right\}, \text { and } C_{j}^{0} \stackrel{d f}{=} C_{K}
$$

and $\left|C_{j}^{r-1}\right|$ is the cardinality (number of elements) of $C_{j}^{r-1}$ [25]. For simplicity, let us assume that $C_{U}=\left\{c_{1}, \ldots, c_{k}\right\}$ and $C_{K}=\left\{c_{k+1}, \ldots, c_{n}\right\}$. It turns out that the iterative procedure proposed in [25] follows the Jacobi iterative method for solving a linear equation system in the form 1 :

$$
A \mu=b
$$

where the matrix $A$ is given as:

$$
A=\left[\begin{array}{cccc}
1 & -\frac{1}{n-1} m_{1,2} & \cdots & -\frac{1}{n-1} m_{1, k} \\
-\frac{1}{n-1} m_{2,1} & 1 & \cdots & -\frac{1}{n-1} m_{2, k} \\
\vdots & \vdots & \vdots & \vdots \\
-\frac{1}{n-1} m_{k-1,1} & \ldots & \ddots & -\frac{1}{n-1} m_{k-1, k} \\
-\frac{1}{n-1} m_{k, 1} & \cdots & -\frac{1}{n-1} m_{k, k-1} & 1
\end{array}\right]
$$

\footnotetext{
${ }^{1}$ The form of the linear equation system (8) is more thoroughly explained in Appendix C
} 
vector of constant terms is

$$
b=\left[\begin{array}{c}
\frac{1}{n-1} m_{1, k+1} \mu\left(c_{k+1}\right)+\ldots+\frac{1}{n-1} m_{1, n} \mu\left(c_{n}\right) \\
\frac{1}{n-1} m_{2, k+1} \mu\left(c_{k+1}\right)+\ldots+\frac{1}{n-1} m_{2, n} \mu\left(c_{n}\right) \\
\vdots \\
\frac{1}{n-1} m_{k, k+1} \mu\left(c_{k+1}\right)+\ldots+\frac{1}{n-1} m_{k, n} \mu\left(c_{n}\right)
\end{array}\right]
$$

and values that need to be determined are denoted as:

$$
\mu^{T}=\left[\mu\left(c_{1}\right), \ldots, \mu\left(c_{k}\right)\right]
$$

The iteration matrix of the Jacobi method is given by:

$$
B_{J}=D^{-1}(E+F)=I-D^{-1} A
$$

The matrix $D$ is the diagonal matrix of the diagonal entries of $A$, hence $D=D^{-1}=I$, whilst $E$ is the lower triangular matrix of entries $e_{i j}=-\left(-\frac{1}{n-1} m_{i, j}\right)=-a_{i j}$, and $F$ is the upper triangular matrix of entries $f_{i j}=-\left(-\frac{1}{n-1} m_{i, j}\right)=-a_{i j}$. Therefore, the update equation (6) can be written in the form:

$$
\mu_{r}\left(c_{i}\right)=\frac{1}{a_{i i}}\left[b_{i}-\sum_{j=1, j \neq i}^{k} a_{i j} \mu_{r-1}\left(c_{j}\right)\right]=b_{i}+\sum_{j=1, j \neq i}^{k} \frac{1}{n-1} m_{i j} \mu_{r-1}\left(c_{j}\right)
$$

When the matrix $A$ is strictly diagonally dominant by rows i.e. $\left|a_{i i}\right|>\Sigma_{j=1}^{n}\left|a_{i j}\right|$ for $i \neq j$ and $i=1, \ldots, k$ then the Jacobi method is convergent [29]2. In our case $a_{i i}=1$ for $i=1, \ldots, k$, hence the $H R E$ procedure is convergent if

$$
1>\sum_{j=1, j \neq i}^{k}\left|a_{i j}\right|
$$

for all $i=1, \ldots, k$. Bearing in mind that $a_{i j}=-\frac{1}{n-1} m_{i j}$ let us note the $H R E$ algorithm has a high chance to be convergent if the set $C_{U}$ is relatively small ( $C_{K}$ is relatively large) and $m_{i j}$ are not too large i.e. estimated values $\mu\left(c_{j}\right)$ for $j=1, \ldots, k$ are similar. Both of these conditions are intuitive and, in practice, are likely to be satisfied. The first of them reflects the natural desire to provide the experts with rather more than the lower number of known reference concepts. The second corresponds to the common-sense observation that all the considered concepts should be similar to each other, because then it is easy to compare them.

The equation (8) could also be solved using direct methods. In such a case it has exactly one solution, if the determinant of $A$ differs from 0, i.e.:

$$
\operatorname{det}(A) \neq 0
$$

Unfortunately, it may turn out that this unique solution $\mu=\left(\mu\left(c_{1}\right), \ldots, \mu\left(c_{k}\right)\right)$ is not in $\mathbb{R}_{+}^{k}$. For instance, some values $\mu\left(c_{i}\right)$ may be less than or equal 0 . In such a case the iterative approach is not convergent (assuming that $\mu\left(c_{j}\right)$ for $c_{j} \in C_{K}$ are strictly positive, the values $\mu\left(c_{i}\right)$ for $i=1, \ldots, k$ must also be strictly positive ${ }^{3}$ ). In such a case $\mu$ that meets (8) cannot be adopted as the $H R E$ procedure output. Instead, the $H R E$ procedure needs to be iterated a predetermined number of times and the result $\mu$ needs to be chosen following the minimizing estimation error heuristics (Sec. 3.2). In the presented approach, only those $m_{j i}$ are determined by experts for which at least one of the two $c_{i}, c_{j}$ comes from $C_{U}$. For two initially known concepts the value $m_{j i}$ is just defined as $m_{j i}=\mu\left(c_{j}\right) / \mu\left(c_{i}\right)$. Hence, the matrix $M$ is always consistent in the part relating to the known concepts i.e. $\forall c_{i}, c_{j} \in C_{K}: m_{j i} \mu\left(c_{i}\right)=\mu\left(c_{j}\right)$.

When the $\mu$ values are not initially known for any $c \in C$, i.e. $C_{K}=\emptyset$, then for an arbitrarily selected $c_{i}$ the value $\mu\left(c_{i}\right)$ might be set by the experimenter to 1 . In such a case the HRE procedure computes the relative order $\mu$ of concepts from $C$ assuming that the weight $\mu\left(c_{i}\right)$ is a unit. Since $c_{i}$ is treated as

\footnotetext{
${ }^{2}$ Note that the Jacobi method is convergent also for $A$ strictly dominant by columns [1]

${ }^{3}$ Note that all the components of the right side of 13 are strictly positive.
} 
the reference element it must be selected with special care. Their relationship with other concepts have a reference meaning, hence they should be highly reliable and well documented.

The final weight vector $\mu_{H R E}$ is synthesized by using $k$ values determined by solving (8) and $n-k$ initially known reference values of concepts from $C_{K}$.

$$
\mu_{H R E}=\left[\mu\left(c_{1}\right), \ldots \mu\left(c_{k}\right), \mu\left(c_{k+1}\right), \ldots, \mu\left(c_{n}\right)\right]^{T}
$$

Thus the rescaled form of $\mu_{H R E}$ is:

$$
\mu_{H R E n}=\left[\frac{\mu\left(c_{1}\right)}{s_{H R E}}, \ldots, \frac{\mu\left(c_{n}\right)}{s_{H R E}}\right] \text { where } s_{H R E}=\sum_{i=1}^{n} \mu\left(c_{i}\right)
$$

\subsection{Heuristics of minimizing estimation error}

The minimizing estimation error heuristics is proposed to deal with the case when it is impossible to uniquely determine $\mu\left(c_{i}\right)$ as the mean of $m_{i j} \mu\left(c_{j}\right)$ for $i \neq j$ (the vector $\mu$ cannot be determined by solving (8) (8). In tests, it was noticed that the more often it happens, the higher the inconsistency. In such a case, rather than solving (8) someone may try to find $\mu$ that minimizes the average absolute estimation error, given as follows:

$$
\widehat{e}_{\mu}=\frac{1}{\left|C_{U}\right|} \sum_{c \in C_{U}} e_{\mu}(c)
$$

where

$$
e_{\mu}\left(c_{j}\right)=\frac{1}{\left|C_{j}^{r-1}\right|} \sum_{c_{i} \in C_{j}^{r-1}}\left|\mu\left(c_{j}\right)-\mu\left(c_{i}\right) \cdot m_{j i}\right|
$$

The problem of minimizing $\widehat{e}_{\mu}$ is discussed in (Appendix $\mathrm{A}$ ). The preliminary Monte Carlo tests show that for the relatively small inconsistency (small $\mathscr{K}$ ) both: the minimizing estimation error heuristic (as defined above) and the averaging with respect to the reference values heuristic (Sec. 3.1) lead to very similar vectors $\mu$. When the inconsistency index $\mathscr{K}$ rises then the solutions provided by these two heuristics become increasingly different. In general, it seems that the heuristic of averaging with respect to the reference values is more useful in practice. However, when the equation (8) does not have an admissible solution and there is an admissible $\mu$ minimizing (18), then the minimizing estimation error heuristic may be worth considering.

Certainly the search for the smallest $\widehat{e}_{\mu}$ makes sense if both: solving (8) and finding $\mu$, which minimizes (18) fail. Then the intermediate $H R E$ iteration result with the minimal absolute estimation error $\widehat{e}_{\mu_{r}}$ needs to be adopted as the output $\mu_{\text {out }}$ of the $H R E$ procedure:

$$
\mu_{\text {out }}=\left\{\mu_{q}: \widehat{e}_{\mu_{q}}=\min \left\{\widehat{e}_{\mu_{1}}, \ldots, \widehat{e}_{\mu_{r}}\right\}\right\}
$$

Although $r$ - the total number of iterations has to be arbitrarily set by an experimenter, in practice, it should be small enough (even one or two iterations may be useful).

\subsection{Heuristics of reciprocity restoration}

According to these heuristics, the input $P C$ matrix $M$ should be reciprocal to be processed by the HRE procedure. Hence, it should hold that $m_{i j}=\frac{1}{m_{j i}}$ for every two ratios $m_{i j}$ and $m_{j i}$ in $M$. Therefore, if the matrix $M$ is not reciprocal, it should be transformed to a similar but reciprocal matrix. Let $\widehat{M}=\left[\widehat{m}_{i j}\right]$ be the new $P C$ matrix obtained from $M=\left[m_{i j}\right]$ by replacing entry $m_{i j}$ in $M$ by the geometric mean of this entry and its (possibly reciprocal) counterpart i.e. $\widehat{m}_{i j}=\left(m_{i j} \frac{1}{m_{j i}}\right)^{1 / 2}$. It is easy to check that the new matrix $\widehat{M}$ is reciprocal. Moreover, if $M$ is initially reciprocal then $\widehat{M}=M$. Therefore, every $P C$ matrix $M$ calculated by the HRE procedure should be preprocessed in order to restore the lost reciprocity property. If $M$ is reciprocal the preprocessed matrix should be identical to $M$. If not, it is recommended to transform $M$ into $\widehat{M}$ according to the definition given above. A similar approach to the lack of the reciprocity property has been discussed, for example, in [13]. The geometric mean properties have been discussed in [10].

\footnotetext{
${ }^{4}$ For the purpose of the $H R E$ approach only $\mu \in \mathbb{R}_{+}^{k}$ are admissible.
} 


\subsection{Heuristics of missing data}

Sometimes there may be a situation that not all indispensable ratios are defined. Then the resulting pairwise comparisons matrix $M$ is incomplete and contains unknown values. In such a case the update equation (6) cannot include the products $m_{j i} \mu\left(c_{i}\right)$ where $m_{j i}$ is not specified. Let us denote $m_{j i}=$ ? if $m_{j i}$ is unspecified.

To handle this situation, the set of elements for which the $\mu_{r-1}$ values were known needs to be changed as follows:

$$
C_{j}^{r-1}=\left\{c \in C: \mu_{r-1}(c) \text { is known, } c \neq c_{j} \text { and } c \neq c_{i} \text { when } m_{j i}=?\right\}
$$

Although incomplete, $M$ should be reciprocal. Hence the reciprocity restoration procedure needs to be extended to the case when some ratios are unknown. Thus, let us define:

$$
\widehat{m}_{i j}= \begin{cases}\left(m_{i j} \frac{1}{m_{j i}}\right)^{1 / 2} & \text { where } m_{j i} \text { and } m_{i j} \text { are specified in } M \\ m_{i j} & \text { where } m_{j i} \text { is unspecified in } M \\ \frac{1}{m_{j i}} & \text { where } m_{i j} \text { is unspecified in } M \\ ? & \text { where } m_{j i} \text { and } m_{i j} \text { are unspecified in } M\end{cases}
$$

The HRE algorithm equipped with the heuristics of missing data can handle matrices to which other methods might not be applicabl 5 . The only limitation is the reachability of the unknown concepts understood as the condition that for each unknown concept $c_{j} \in C_{U}$ there must exist at least one concept $c_{i} \in C_{K}$ with known weight and a sequence of indices $i_{1}, i_{2}, \ldots, i_{q}$ such that $m_{i i_{1}} \neq ?, m_{i_{1} i_{2}} \neq$ ? $, \ldots, m_{i_{q} j} \neq$ ?, where $i_{1}, i_{2}, \ldots, i_{q} \in\{1,2, \ldots, n\}$. Therefore, the $H R E$ procedure is able to propose the value $\mu_{q}\left(c_{j}\right)$ for $c_{j} \in C_{U}$ only if there is at least one $c_{r} \in C_{U}$ for which the product $m_{j, r} \mu_{q-1}\left(c_{r}\right)$ is known. In the case of an incomplete matrix the weights cannot be obtained by solving a linear equation system as shown in (8). In particular, due to the missing data, the set of known values $C_{j}^{r-1}$ for $j$ such that $c_{j} \in C_{U}$ may change for the second and subsequent iteration. Thus, the incomplete data requires an iterative approach when every subsequent value of $\mu\left(c_{j}\right)$ is estimated according to the update rule (6). If the procedure converges, a sufficiently accurate approximation might be adopted as the output. If not, the one with the smallest $\widehat{e}_{\mu}$ from the several initial iteration results needs to be adopted as the result of the procedure.

The missing data heuristics might be especially useful when a large number of different concepts should be compared with each other. In such a case the completion of all the ratios in the matrix $M$ might be difficult, which may result in its incompleteness.

\section{Numerical examples}

Despite the fact that in the HRE approach the priorities of some concepts have to be initially known, the procedure might be used (for caution) in any case. However, this will require the adoption of arbitrarily selected elements as the reference concepts. The first numerical example (from [2]) demonstrates the case when the standard $P C$ matrix is processed by the $H R E$ algorithm and the arbitrary concept is chosen as the reference one. The second example shows a typical situation for $H R E$. There is a non-empty set $C_{K}$ of the reference concepts and the set $C_{U}$ consists of unknown elements. The third example addresses the problem of non-reciprocal matrices and demonstrates how the heuristic of reciprocity restoration works in practice. The last, fourth, example deals with an incomplete $P C$ matrix. It uses an iterative version of the $H R E$ procedure to derive the weight vector from $M$. It is designed to demonstrate how the iterative $H R E$ procedure equipped with the missing data heuristic may support incomplete $P C$ data sets.

\section{Example 1 (Case of verbal judgements)}

Let $c_{1}, \ldots, c_{5}$ be a set of concepts for which the following judgements were formulated by a person $J: c_{1}$ equally to moderately dominates $c_{2}, c_{1}$ moderately dominates $c_{3}, c_{1}$ strongly dominates $c_{4}, c_{1}$ extremely dominates

\footnotetext{
${ }^{5}$ Various methods have their extensions to enable them to handle such cases, for example, the LSM extension can be found in [5].
} 
$c_{5}, c_{2}$ equally to moderately dominates $c_{3}, c_{2}$ moderately to strongly dominates $c_{4}, c_{2}$ extremely dominates $c_{5}, c_{3}$ equally to moderately dominates $c_{4}, c_{3}$ very strongly dominates $c_{5}, c_{4}$ very strongly dominates $c_{5}$. Then, adopting the method of converting verbal judgements into numbers proposed in [32] the following $P C$ matrix is obtained:

$$
M=\left[\begin{array}{lllll}
1 & 2 & 3 & 5 & 9 \\
\frac{1}{2} & 1 & 2 & 4 & 9 \\
\frac{1}{3} & \frac{1}{2} & 1 & 2 & 8 \\
\frac{1}{5} & \frac{1}{4} & \frac{1}{2} & 1 & 7 \\
\frac{1}{9} & \frac{1}{9} & \frac{1}{8} & \frac{1}{7} & 1
\end{array}\right]
$$

The rescaled eigenvector $\mu_{E V}$ (see 3) corresponding to the maximal eigenvalue of $M$ is:

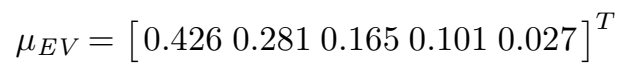

The geometric mean based weight vector (see 4) for $M$ is:

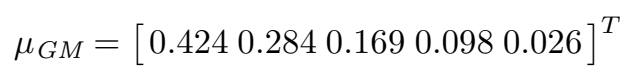

As reported in 2 the ranking $\mu_{E V}$ does not meet the Condition of Order Preservation (herein abbreviated as $C O P$, see Appendix (B). In particular, since the value $m_{1,4}=4$ is smaller than $m_{4,5}=7$, COP also requires that $\frac{\mu_{E V}\left(c_{1}\right)}{\mu_{E V}\left(c_{4}\right)}<\frac{\mu_{E V}\left(c_{4}\right)}{\mu_{E V}\left(c_{5}\right)}$. It is easy to calculate that $\frac{\mu_{E V}\left(c_{1}\right)}{\mu_{E V}\left(c_{4}\right)}=4.218$ and $\frac{\mu_{E V}\left(c_{4}\right)}{\mu_{E V}\left(c_{5}\right)}=3.741$ which is in contradiction with the second $C O P$ postulate (58). It is easy to check that for $\mu_{G M} C O P$ does not hold either. The eigenvalue based inconsistency index is low and equals $C I=0.057$. In contrast, Koczkodaj's distance based inconsistency index is high 6 and equals $\mathscr{K}(M)=0.743$.

To calculate the rank using the $H R E$ approach when none of the concepts are initially known (i.e. $C_{K}=\emptyset$ ), it is necessary to choose some $c \in C_{U}$ and assign an arbitrary weight to it. Thus, based on our knowledge about the problem domain, let us assume that $c_{1}$ is a reference element $\left(C_{K}=\left\{c_{1}\right\}\right.$ and $\left.C_{U}=C_{U} \backslash\left\{c_{1}\right\}\right)$ and set $\mu\left(c_{1}\right)=1$. (It is easy to check that for a rescaled form of a weight vector $\mu$ the exact value assigned to $\mu\left(c_{1}\right)$ is not important). Then, after the first $H R E$ iteration, the matrix $A$ and vector $b$ are determined 7 ,

$$
A=\left[\begin{array}{cccc}
1 & -\frac{1}{n-1} m_{2,3} & -\frac{1}{n-1} m_{2,4}-\frac{1}{n-1} m_{2,5} \\
-\frac{1}{n-1} m_{3,2} & 1 & -\frac{1}{n-1} m_{3,4} & -\frac{1}{n-1} m_{3,5} \\
-\frac{1}{n-1} m_{4,2} & -\frac{1}{n-1} m_{4,3} & 1 & -\frac{1}{n-1} m_{4,5} \\
-\frac{1}{n-1} m_{5,2} & -\frac{1}{n-1} m_{5,3} & -\frac{1}{n-1} m_{5,4} & 1
\end{array}\right], \quad b=\left[\begin{array}{c}
\frac{1}{n-1} m_{2,1} \mu\left(c_{1}\right) \\
\frac{1}{n-1} m_{3,1} \mu\left(c_{1}\right) \\
\frac{1}{n-1} m_{4,1} \mu\left(c_{1}\right) \\
\frac{1}{n-1} m_{5,1} \mu\left(c_{1}\right)
\end{array}\right]
$$

so that the equation (8) takes the form:

$$
\left[\begin{array}{cccc}
1 & -0.5 & -1 & -2.25 \\
-0.125 & 1 & -0.5 & -2 \\
-0.062 & -0.125 & 1 & -1.75 \\
-0.028 & -0.031 & -0.036 & 1
\end{array}\right]\left[\begin{array}{l}
\mu\left(c_{2}\right) \\
\mu\left(c_{3}\right) \\
\mu\left(c_{4}\right) \\
\mu\left(c_{5}\right)
\end{array}\right]=\left[\begin{array}{c}
0.125 \\
0.083 \\
0.05 \\
0.028
\end{array}\right]
$$

(Note that $\left|C_{U}\right|=4$ implies that the dimensions of matrix $A$ are $4 \times 4$ ). Since $\operatorname{det}(A) \neq 0$ and $\mu\left(c_{i}\right)>0$ for $i=2, \ldots, 5$ then the rescaled vector $\mu_{H R E n}$ obtained by solving (8) is adopted as the output of the HRE algorithm (an iterative procedure leads to the same solution).

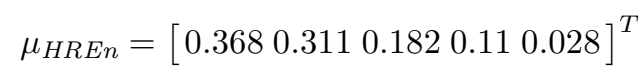

By examining all the possible cases, it is easy to check that the weight vector $\mu_{H R E n}$ satisfies $C O P$. It is noteworthy that all the three vectors: $\mu_{E V}, \mu_{G M}$ and $\mu_{H R E n}$ preserve the same order of elements and they differ only in intensities of preferences. Since the value $\mu\left(c_{1}\right)$ is chosen arbitrarily by an experimenter, the

\footnotetext{
${ }^{6}$ The work [24] suggests that an acceptable threshold of inconsistency $\mathscr{K}(M)$, for most practical applications, turns out to be $1 / 3$.

${ }^{7}$ In practice, the matrix $A$ can be obtained from the matrix $M$ by removing the rows and columns corresponding to concepts from $C_{K}$, and multiplying the remaining values (except diagonal) by $-1 /(n-1)$. The removed rows and columns form the vector $b$ as shown in (10).
} 
obtained result has only an ordinal meaning. As both vectors $\mu_{H R E n}$ and $\mu_{H R E}$ carry the same (ordinal) information it is convenient to consider the rescaled vector $\mu_{H R E n}$.

\section{Example 2 (Case with reference concept values)}

The immediate inspiration for the second example is the scientific units evaluation in Poland. The proposed ranking algorithm [28] is based on the pairwise comparisons paradigm although it does not follow the $A H P$ approach. The reference scientific units (as defined therein) are used to determine the scientific categories, and thereby funding levels.

Let $c_{1}, \ldots, c_{5}$ represent the hypothetical scientific units, where two of them $c_{2}$ and $c_{3}$ are the reference units for which the values $c_{2}, c_{3} \in C_{K}$ are initially known and equal $\mu\left(c_{2}\right)=5$ and $\mu\left(c_{3}\right)=7$. The analysis of the scientific units $c_{1}, c_{4}$ and $c_{5}$ with respect to the criterion $\mu$ allows the formulation of the following pairwise comparisons matrix:

$$
M=\left[\begin{array}{ccccc}
1 & \frac{3}{5} & \frac{4}{7} & \frac{5}{8} & \frac{1}{2} \\
\frac{5}{3} & 1 & \frac{5}{7} & \frac{5}{2} & \frac{10}{3} \\
\frac{7}{4} & \frac{7}{5} & 1 & \frac{7}{2} & 4 \\
\frac{8}{5} & \frac{2}{5} & \frac{2}{7} & 1 & \frac{4}{3} \\
2 & \frac{3}{10} & \frac{1}{4} & \frac{3}{4} & 1
\end{array}\right]
$$

The rescaled eigenvector $\mu_{E V}$ (see 3) and the rescaled geometric mean based vector $\mu_{G M}$ (see 4) for $M$ are as follows:

$$
\mu_{E V}=\left[\begin{array}{lllll}
0.12 & 0.275 & 0.356 & 0.131 & 0.118
\end{array}\right]^{T}
$$

and

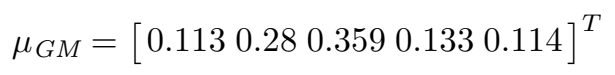

The HRE approach requires the solution of the linear equation system for $A$ and $b$ as follow: 8 :

$$
A=\left[\begin{array}{ccc}
1 & -\frac{1}{n-1} m_{1,4}-\frac{1}{n-1} m_{1,5} \\
-\frac{1}{n-1} m_{4,1} & 1 & -\frac{1}{n-1} m_{4,5} \\
-\frac{1}{n-1} m_{5,1}-\frac{1}{n-1} m_{5,4} & 1
\end{array}\right], \quad b=\left[\begin{array}{l}
\frac{1}{n-1} m_{1,2} \mu\left(c_{2}\right)+\frac{1}{n-1} m_{1,3} \mu\left(c_{3}\right) \\
\frac{1}{n-1} m_{4,2} \mu\left(c_{2}\right)+\frac{1}{n-1} m_{4,3} \mu\left(c_{3}\right) \\
\frac{1}{n-1} m_{5,2} \mu\left(c_{2}\right)+\frac{1}{n-1} m_{5,3} \mu\left(c_{3}\right)
\end{array}\right]
$$

hence, numerically:

$$
\left[\begin{array}{ccc}
1 & -0.156 & -0.125 \\
-0.4 & 1 & -0.333 \\
-0.5 & -0.187 & 1
\end{array}\right]\left[\begin{array}{l}
\mu\left(c_{1}\right) \\
\mu\left(c_{4}\right) \\
\mu\left(c_{5}\right)
\end{array}\right]=\left[\begin{array}{c}
1.75 \\
1.0 \\
0.812
\end{array}\right]
$$

The not rescaled $\mu_{H R E}$ weight vector is:

$$
\mu_{H R E}=\left[\begin{array}{lll}
2.5275 .07 .02 .88 & 2.616
\end{array}\right]^{T}
$$

and after rescaling:

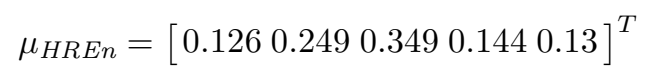

The inconsistency indices are $C I=0.07$ (AHP) and $\mathscr{K}(M)=0.781$ (Koczkodaj).

It is easy to observe that in this hypothetical case the eigenvalue vector $\mu_{E V}$ also violates $C O P$. That is because the ratio $m_{1,5}=\frac{1}{2}<1$, whilst $\frac{\mu_{E V}\left(c_{1}\right)}{\mu_{E V}\left(c_{5}\right)}>1$. The $\mu_{G M}$ and $\mu_{H R E}$ do not violate the first $C O P$ postulate (Appendix B). However, all the vectors $\mu_{E V}, \mu_{G M}$ and $\mu_{H R E}$ do not meet the second COP postulate.

\footnotetext{
${ }^{8}$ note that $\left|C_{U}\right|=3$ implies that the dimensions of matrix $A$ are $3 \times 3$
} 


\section{Example 3 (Case of not reciprocal matrix)}

The third example concerns a situation when the $P C$ matrix is almost consistent but not reciprocal. Due to the lack of reciprocity, the use of the eigenvalue method as well as the geometric means method might be disputed (these methods are designed for reciprocal matrices [19]). Hence, the values $\mu_{E V}$ and $\mu_{G M}$ are computed just for testing the robustness and sensitivity of both methods to the incorrect data.

Let $c_{1}, \ldots, c_{4}$ represent four candidates for the position of a manager in some production company. As different examiners have been involved in the recruitment process, one examiner rated $c_{4}$ social skills twice as high as $c_{1}$, whilst another examiner, while comparing skills $c_{1}$ to $c_{4}$, ruled that both candidates are exactly on the same level. Assuming that in all other cases the recruitment committee has ruled that all other candidates present the same level of social skills, the $P C$ matrix $M$ representing the problem may appear as follows:

$$
M=\left[\begin{array}{llll}
1 & 1 & 1 & 1 \\
1 & 1 & 1 & 1 \\
1 & 1 & 1 & 1 \\
2 & 1 & 1 & 1
\end{array}\right]
$$

An attempt to calculate the eigenvector based or geometric mean based rank leads to the following vectors:

$$
\begin{aligned}
& \mu_{E V}=\left[\begin{array}{llll}
0.236 & 0.236 & 0.236 & 0.292
\end{array}\right]^{T} \\
& \mu_{G M}=\left[\begin{array}{llll}
0.239 & 0.239 & 0.239 & 0.284
\end{array}\right]^{T}
\end{aligned}
$$

For the purpose of the $H R E$ algorithm the reciprocity property of $M$ must be restored. Thus, according to the heuristics of reciprocity restoration $M$ is transformed to $\widehat{M}$ in the form:

$$
\widehat{M}=\left[\begin{array}{cccc}
1 & 1 & 1 & 0.707 \\
1 & 1 & 1 & 1 \\
1 & 1 & 1 & 1 \\
1.414 & 1 & 1 & 1
\end{array}\right]
$$

then $\widehat{M}$ is processed following procedures formulated in (Sec. 3.1 and 3.2). Since $C_{k}$ cannot be empty, then let us adopt $c_{1}$ as the reference element i.e. $C_{K}=C_{K} \cup\left\{c_{1}\right\}$ and $\mu\left(c_{1}\right)=1$. Then, the matrix $A$ and vector $b$ can be determined,

$$
A=\left[\begin{array}{ccc}
1 & -\frac{1}{n-1} \widehat{m}_{2,3}-\frac{1}{n-1} \widehat{m}_{2,4} \\
-\frac{1}{n-1} \widehat{m}_{3,2} & 1 & -\frac{1}{n-1} \widehat{m}_{3,4} \\
-\frac{1}{n-1} \widehat{m}_{4,2}-\frac{1}{n-1} \widehat{m}_{4,3} & 1
\end{array}\right], \quad b=\left[\begin{array}{c}
\frac{1}{n-1} \widehat{m}_{2,1} \mu\left(c_{1}\right) \\
\frac{1}{n-1} \widehat{m}_{3,1} \mu\left(c_{1}\right) \\
\frac{1}{n-1} \widehat{m}_{4,1} \mu\left(c_{1}\right)
\end{array}\right]
$$

thus, to determine the vector $\mu_{H R E}$ the following linear equation system needs to be solved:

$$
\left[\begin{array}{ccc}
1 & -0.333 & -0.333 \\
-0.333 & 1 & -0.333 \\
-0.333 & -0.333 & 1
\end{array}\right]\left[\begin{array}{l}
\mu\left(c_{2}\right) \\
\mu\left(c_{3}\right) \\
\mu\left(c_{4}\right)
\end{array}\right]=\left[\begin{array}{l}
0.333 \\
0.333 \\
0.471
\end{array}\right]
$$

The rescaled $H R E$ weight vector is:

$$
\mu_{H R E n}=\left[\begin{array}{llll}
0.227 & 0.25 & 0.25 & 0.273
\end{array}\right]^{T}
$$

Although all the tested methods rate the $c_{4}$ candidate higher than the others, only the $H R E$ method rates $c_{1}$ below the average. Hence, only the $H R E$ algorithm meets $C O P$, i.e. $m_{4,1}>m_{4,2} \Rightarrow \frac{\mu\left(c_{4}\right)}{\mu\left(c_{1}\right)}>\frac{\mu\left(c_{4}\right)}{\mu\left(c_{2}\right)}$ is met only by $\mu_{H R E}$ (let us note that $\mu_{E V}\left(c_{1}\right)=\mu_{E V}\left(c_{2}\right)$ as well as $\mu_{G M}\left(c_{1}\right)=\mu_{G M}\left(c_{2}\right)$, thus $\mu_{E V}\left(c_{4}\right) / \mu_{E V}\left(c_{1}\right)=$ $\mu_{E V}\left(c_{4}\right) / \mu_{E V}\left(c_{2}\right)$ and $\left.\mu_{G M}\left(c_{4}\right) / \mu_{G M}\left(c_{1}\right)=\mu_{G M}\left(c_{4}\right) / \mu_{G M}\left(c_{2}\right)\right)$. Although the eigenvalue and geometric mean methods have a problem with $C O P$ when $M$ is not reciprocal, it should be noted that they have no problems with $C O P$ for $\widehat{M}$. This may suggest that the reciprocity restoration heuristic might be useful also for other weight derivation methods. 


\section{Example 4 (Case of incomplete matrix)}

The fourth example represents situations where some data are missing. The known ratios representing the relative importance of concepts were placed into the matrix $M$. Question marks at the intersection of row $i$ and column $j$ in the matrix (43) mean unknown values $m_{i j}$. The immediate inspiration for this example was an observation of the meta analysis process in biochemistry [4] where the number and diversity of analyzed factors make drawing the final conclusions difficult or even impossible.

Let us consider the four drugs $c_{1}, \ldots, c_{4}$ with proven efficacy in controlling the disease $X$. Based on the available scientific articles, Dr H. came to the conclusion that $c_{1}$ and $c_{2}$ have similar efficacy, the same for $c_{3}$ and $c_{4}$. He also came across research showing that in some cases $c_{2}$ is two times more effective than $c_{3}$, and also $c_{4}$ fails three times more likely than $c_{1}$. Unfortunately, Dr. H. found no studies comparing the therapeutic effect of drugs in pairs $\left(c_{1}, c_{3}\right)$ and $\left(c_{2}, c_{4}\right)$. Therefore the $P C$ matrix $M$ (43) prepared by Dr $\mathrm{H}$. looks like as follows:

$$
\mathrm{M}=\left[\begin{array}{llll}
1 & 1 & ? & ? \\
? & 1 & 2 & ? \\
? & ? & 1 & ? \\
\frac{1}{3} & ? & 1 & 1
\end{array}\right]
$$

The drug $c_{1}$ is very popular, so there are many studies on its efficacy. There are also some studies that compare efficacy of $c_{1}$ and $c_{2}$ but $c_{2}$ is less popular. Since $c_{1}$ is the most popular drug on $X$, and what follows, the relationship between $c_{1}$ and $c_{2}$ have been most extensively tested, then $c_{1}$ has been adopted as the reference concept, i.e. $C_{K}=C_{K} \cup\left\{c_{1}\right\}$ and $\mu\left(c_{1}\right)=1$. The HRE procedure, applied to $M$ (a reciprocity restoration included) converges to:

$$
\mu_{H R E n}=\left[\begin{array}{llll}
0.369 & 0.338 & 0.154 & 0.138
\end{array}\right]^{T}
$$

Thus, the most recommended cure for $\mathrm{X}$ is $c_{1}$, then $c_{2}, c_{3}$ and $c_{4}$. It should be noted that the proposed weights by the HRE algorithm are in line with $C O P$. For example, if $m_{2,3}=2>1$ then also $\frac{\mu_{H R E}\left(c_{1}\right)}{\mu_{H R E}\left(c_{3}\right)}=$ $2,396>1$, Similarly, $m_{4,1}=\frac{1}{3}<1$ then also $\frac{\mu_{H R E}\left(c_{4}\right)}{\mu_{H R E}\left(c_{1}\right)}=0,374<1$ etc. Due to the incompatible input matrix format, the eigenvalue method and the geometric mean method could not be used in this case9.

\section{Summary}

The quality of the results achieved using the HRE approach is inextricably linked to input data quality. According to the popular adage "garbage in, garbage out", when data are bad even the best algorithm is not able to provide good output. In the case of heuristic algorithms, the domain of applicability depends on the adopted heuristics. Despite the promising results for different types (and different quality) of input data, the application area of the HRE approach has only been sketched. It is therefore necessary to conduct further research to better define assumed heuristics and the situations in which they may be most useful. In particular, relationships between different formulations of data inconsistency levels and the priority estimation quality seem to be very interesting.

The HRE approach presented in the article is based on the iterative $H R E$ algorithm primarily formulated in [25. The heuristics indicated are much more thoroughly analyzed in this work. In particular, the heuristic of averaging with respect to the reference value and the heuristic of minimizing estimation error are given in the general form as the linear equation system solving problems. The new useful heuristic of reciprocity restoration has been introduced and the incomplete $P C$ matrix problem has been addressed. The presented theoretical considerations are accompanied by four numerical examples demonstrating different situations in which the proposed solution might be helpful. The HRE approach tries to complement other methods. It has been designed to help estimation of the relative order of concepts when a non-empty reference subset of concepts is known (or a set of such can be readily determined). Therefore, with this new application area, it may be of interest to a wide range of both researchers and practitioners.

\footnotetext{
${ }^{9}$ There are several approaches that address the problem of incomplete PC data. See e.g. 6 6 14]22
} 


\section{Acknowledgements}

I would like to thank Dr Jarosław Wąs for reading the first version of this work, his comments and corrections. I am also grateful to Prof. Antoni Ligezza for valuable discussions and constant support and encouragement. Special thanks are due to Ian Corkill for his editorial help.

\section{References}

1. R. Bagnara. A unified proof for the convergence of jacobi and gauss-seidel methods. SIAM Review, $37,1995$.

2. C. A. Bana e Costa and J. Vansnick. A critical analysis of the eigenvalue method used to derive priorities in AHP. European Journal of Operational Research, 187(3):1422-1428, June 2008.

3. J. Barzilai and B. Golany. AHP rank reversal, normalization and aggregation rules. INFOR - Information Systems and Operational Research, 32(2):57-64, 1994.

4. A. Bodzoń-Kułakowska, K. Kułakowski, A. Drabik, A. Moszczynski, J. Silberring, and P. Suder. Morphinome-a meta-analysis applied to proteomics studies in morphine dependence. Proteomics, 11(1):5-21, January 2011.

5. S. Bozóki. Solution of the least squares method problem of pairwise comparison matrices. Central European Journal of Operations Research, 16(4):345-358, 2008.

6. S. Bozóki, J. Fülöp, and L. Rónyai. On optimal completion of incomplete pairwise comparison matrices. Mathematical and Computer Modelling, 52(1-2):318 - 333, 2010.

7. S. Bozóki and T. Rapcsak. On Saaty's and Koczkodaj's inconsistencies of pairwise comparison matrices. Journal of Global Optimization, 42(2):157-175, 2008.

8. M. Brunelli, L. Canal, and M. Fedrizzi. Inconsistency indices for pairwise comparison matrices: a numerical study. Annals of Operations Research, February 2013.

9. J. M. Colomer. Ramon Llull: from 'Ars electionis' to social choice theory. Social Choice and Welfare, 40(2):317328, October 2011.

10. G. B. Crawford. The geometric mean procedure for estimating the scale of a judgement matrix. Mathematical Modelling, 9(3-5):327 - 334, 1987.

11. J. S. Dyer. Remarks on the analytic hierarchy process. Management Science, 36(3):249-258, 1990.

12. G. T. Fechner. Elements of psychophysics, volume 1. Holt, Rinehart and Winston, New York, 1966.

13. M. Fedrizzi and M. Brunelli. On the priority vector associated with a reciprocal relation and a pairwise comparison matrix. Journal of Soft Computing, 14(6):639-645, January 2010.

14. M. Fedrizzi and S. Giove. Incomplete pairwise comparison and consistency optimization. European Journal of Operational Research, 183(1):303-313, 2007.

15. S. Greco, B. Matarazzo, and R. Słowiński. Dominance-based rough set approach on pairwise comparison tables to decision involving multiple decision makers. In JingTao Yao, Sheela Ramanna, Guoyin Wang, and Zbigniew Suraj, editors, Rough Sets and Knowledge Technology, volume 6954 of Lecture Notes in Computer Science, pages 126-135. Springer Berlin Heidelberg, 2011.

16. William Ho. Integrated analytic hierarchy process and its applications - A literature review. European Journal of Operational Research, 186(1):18-18, March 2008.

17. A. Ishizaka and A. Labib. Analytic hierarchy process and expert choice: Benefits and limitations. OR Insight, 22(4):201-220, 2009.

18. A. Ishizaka and A. Labib. Review of the main developments in the analytic hierarchy process. Expert Systems with Applications, 38(11):14336-14345, October 2011.

19. A. Ishizaka and M. Lusti. How to derive priorities in AHP: a comparative study. Central European Journal of Operations Research, 14(4):387-400, December 2006.

20. R Janicki and Y. Zhai. On a pairwise comparison-based consistent non-numerical ranking. Logic Journal of the IGPL, 20(4):667-676, 2012.

21. W. W. Koczkodaj. A new definition of consistency of pairwise comparisons. Math. Comput. Model., 18(7):79-84, October 1993.

22. W. W. Koczkodaj, M. W. Herman, and M. Orlowski. Managing Null Entries in Pairwise Comparisons. Knowledge and Information Systems, 1(1):119-125, 1999.

23. W. W. Koczkodaj and M. Orłowski. Computing a consistent approximation to a generalized pairwise comparisons matrix. Computers $\&$ Mathematics with Applications, 37(3):79-85, 1999.

24. W. W. Koczkodaj and S. J. Szarek. On distance-based inconsistency reduction algorithms for pairwise comparisons. Logic Journal of the IGPL, 18(6):859-869, October 2010.

25. K. Kułakowski. A heuristic rating estimation algorithm for the pairwise comparisons method. Central European Journal of Operations Research, pages 1-17, 2013.

26. M. J. Liberatore and R. L. Nydick. The analytic hierarchy process in medical and health care decision making: A literature review. European Journal of Operational Research, 189(1):14-14, August 2008. 
27. L. Mikhailov. Deriving priorities from fuzzy pairwise comparison judgements. Fuzzy Sets and Systems, 134(3):365385, March 2003.

28. Ministry of Science and Higher Education. Regulation on principles of science financing (Polish: Rozporządzenie Ministra Nauki i Szkolnictwa Wyższego w sprawie kryteriów i trybu przyznawania kategorii naukowej jednostkom naukowym). Dziennik Ustaw Rzeczypospolitej Polskiej, 877, 2012.

29. A. Quarteroni, R. Sacco, and F. Saleri. Numerical mathematics. Springer Verlag, 2000.

30. T. L. Saaty. A scaling method for priorities in hierarchical structures. Journal of Mathematical Psychology, 15(3):234 - 281, 1977.

31. T. L. Saaty. An Exposition on the AHP in Reply to the Paper "Remarks on the Analytic Hierarchy Process". Management Science, 36(3):259-268, March 1990.

32. T. L. Saaty. The analytic hierarchy and analytic network processes for the measurement of intangible criteria and for decision-making. In Multiple Criteria Decision Analysis: State of the Art Surveys, volume 78 of International Series in Operations Research and Management Science, pages 345-405. Springer New York, 2005.

33. T. L. Saaty. Relative Measurement and Its Generalization in Decision Making. Why Pairwise Comparisons are Central in Mathematics for the Measurement of Intangible Factors. The Analytic Hierarchy/Network Process. Estadística e Investigación Operativa / Statistics and Operations Research (RACSAM), 102:251-318, November 2008.

34. T. L. Saaty and G. Hu. Ranking by eigenvector versus other methods in the analytic hierarchy process. Applied Mathematics Letters, 11(4):121-125, 1998.

35. N. Schlager and J. Lauer, editors. Science and its times: understanding the social significance of scientific discovery, volume 2. Schlager Information Group, 2000.

36. J. E. Smith and D. Von Winterfeldt. Anniversary article: decision analysis in management science. Management Science, 50(5):561-574, 2004.

37. N. Subramanian and R. Ramanathan. A review of applications of Analytic Hierarchy Process in operations management. International Journal of Production Economics, 138(2):215-241, August 2012.

38. L. L. Thurstone. A law of comparative judgment, reprint of an original work published in 1927. Psychological Review, 101:266-270, 1994.

39. O. S. Vaidya and S. Kumar. Analytic hierarchy process: An overview of applications. European Journal of Operational Research, 169(1):1-29, February 2006.

40. K. K. F. Yuen. Analytic hierarchy prioritization process in the AHP application development: A prioritization operator selection approach. Appl. Soft Comput., 10(4):975-989, 2010.

41. K. K. F. Yuen. Membership Maximization Prioritization Methods for Fuzzy Analytic Hierarchy Process. Fuzzy Optimization and Decision Making, 11(2):113-133, June 2012.

42. K. K. F. Yuen. Fuzzy cognitive network process: Comparison with fuzzy analytic hierarchy process in new product development strategy. Fuzzy Systems, IEEE Transactions on, PP(99):1-1, 2013.

\section{A About the heuristic of minimizing estimation error}

From the point of view of the heuristics of minimizing estimation error, the best solution $\mu$ should minimize $\widehat{e}_{\mu}$

$$
\widehat{e}_{\mu}=\frac{1}{\left|C_{U}\right|} \sum_{c \in C_{U}} e_{\mu}(c)
$$

where

$$
e_{\mu}\left(c_{j}\right)=\frac{1}{\left|C_{j}^{r-1}\right|} \sum_{c_{i} \in C_{j}^{r-1}}\left|\mu\left(c_{j}\right)-\mu\left(c_{i}\right) \cdot m_{j i}\right|
$$

The problem by replacing the absolute difference $\left|\mu\left(c_{j}\right)-\mu\left(c_{i}\right) \cdot m_{j i}\right|$ by the squared difference $\left(\mu\left(c_{j}\right)-\right.$ $\left.\mu\left(c_{i}\right) \cdot m_{j i}\right)^{2}$ leads to the equivalent one of finding the $\mu$ minimizing function $f: \mathbb{R}_{+}^{k} \rightarrow \mathbb{R}$ given as:

$$
\begin{aligned}
f\left(\mu\left(c_{1}\right), \ldots, \mu\left(c_{k}\right)\right)=\sum_{j \in I_{U}} \sum_{i \in I_{U} \backslash\{j\}}\left(\mu\left(c_{j}\right)-\mu\left(c_{i}\right) \cdot m_{j i}\right)^{2} & \\
& +\sum_{j \in I_{U}} \sum_{i \in I_{K}}\left(\mu\left(c_{j}\right)-\mu\left(c_{i}\right) \cdot m_{j i}\right)^{2}
\end{aligned}
$$


where $I_{U}, I_{K}$ and $I_{C}$ denote the sets of indices of elements from $C_{U}, C_{K}$ and $C$ correspondingly 10 .

In order to determine the extremum of the function $f$, the following linear equation system needs to be solved:

$$
\left[\begin{array}{c}
\frac{\partial f}{\partial \mu\left(c_{1}\right)} \\
\vdots \\
\frac{\partial f}{\partial \mu\left(c_{k}\right)}
\end{array}\right]=0
$$

where every single equation has the form:

$$
\begin{aligned}
& \frac{\partial f}{\partial \mu\left(c_{j}\right)}=2 \cdot\left(\sum_{i \in I_{U} \backslash\{j\}}\left(\mu\left(c_{j}\right)-\mu\left(c_{i}\right) \cdot m_{j i}\right)-\right. \\
& \left.-\sum_{i \in I_{U} \backslash\{j\}}\left(\mu\left(c_{i}\right)-\mu\left(c_{j}\right) \cdot m_{i j}\right) \cdot m_{i j}+\sum_{i \in I_{K}}\left(\mu\left(c_{j}\right)-\mu\left(c_{i}\right) \cdot m_{j i}\right)\right)=0
\end{aligned}
$$

for $j \in I_{U}$. Hence, the above equation is equivalent to:

$$
\left(n-1+\sum_{i \in I_{U} \backslash\{j\}} m_{i j}^{2}\right) \mu\left(c_{j}\right)-\sum_{i \in I_{U} \backslash\{j\}}\left(m_{j i}+m_{i j}\right) \cdot \mu\left(c_{i}\right)+\sum_{i \in I_{K}} \mu\left(c_{i}\right) \cdot m_{j i}=0
$$

Dividing both sides of (49) by $(n-1)$, and denoting $\frac{1}{n-1} \sum_{i \in I_{U} \backslash\{j\}} m_{i j}^{2} \stackrel{d f}{=} S_{j}$ it is easy to observe that (50) turns into:

$$
\begin{aligned}
& -\frac{m_{j 1}+m_{1 j}}{n-1} \mu\left(c_{1}\right)-\frac{m_{j 2}+}{n-1} m_{2 j} \\
& n
\end{aligned}
$$

Thus, finding the extremum point of $f$ boils down to solving the following equation:

$$
E \mu=b
$$

where:

$$
E=\left[\begin{array}{cccc}
1+S_{1} & -\frac{m_{1,2}+m_{2,1}}{n-1} & \ldots & -\frac{m_{1, k}+m_{k, 1}}{n-1} \\
-\frac{m_{2,1}+m_{1,2}}{n-1} & 1+S_{2} & \cdots & -\frac{m_{2, k}+m_{k, 2}}{n-1} \\
\vdots & \vdots & \vdots & \vdots \\
\frac{m_{k-1,1}+m_{1, k-1}}{n-1} & \ldots & \ddots & -\frac{m_{k-1, k}+m_{k, k-1}}{n-1} \\
-\frac{m_{k, 1}+m_{1, k}}{n-1} & \cdots & -\frac{m_{k, k-1}+m_{k-1, k}}{n-1} & 1+S_{k}
\end{array}\right]
$$

and $b$ is defined as in (10). It is easy to show that the Hessian matrix defined as:

$$
H_{i j}=\left[\frac{\partial^{2} f}{\partial \mu\left(c_{j}\right) \partial \mu\left(c_{i}\right)}\right]
$$

equals:

$$
H=2(n-1) E
$$

Therefore, if $E$ is strictly diagonally dominant, then $H$ is also strictly diagonally dominant. Since the diagonal entries of $H$ are all positive, then $H$ is positively definite [29, page 29]. Thus, for $E$ strictly diagonally dominant the solution of (52) is the minimum of $f$.

$\overline{{ }^{10} \text { In particular }}$ it is assumed that $I_{U}=\{1, \ldots, k\}$ 


\section{B Condition of order preservation}

Among the various criticisms raised at $A H P$ and the eigenvalue method, a Condition of Order Preservation $(C O P)$ postulate [2] seems to be one of the more interesting. According to $C O P$, the output of the weight calculation method should preserve the order as well as the intensity of preferences. In other words, COP is met by the weight vector $\mu$ if for every four concepts $c_{1}, \ldots, c_{4} \in C$ such that $c_{1}$ dominates $c_{2}$ more than $c_{3}$ dominates $c_{4}$ in $M$ i.e. $m_{1,2}>1 \wedge m_{3,4}>1 \wedge m_{1,2}>m_{3,4}$, the following two assertions are true:

1. Preservation of Order of Preference $(P O P)$

$$
\begin{aligned}
& \mu\left(c_{1}\right)>\mu\left(c_{2}\right) \\
& \mu\left(c_{3}\right)>\mu\left(c_{4}\right)
\end{aligned}
$$

2. Preservation of Order of Intensity of Preference $(P O I P)$

$$
\frac{\mu\left(c_{1}\right)}{\mu\left(c_{2}\right)}>\frac{\mu\left(c_{3}\right)}{\mu\left(c_{4}\right)}
$$

COP does not depend on any concepts specific for the eigenvalue method. It reflects the natural desire that the final ranking should be consistent with the individual expert judgments. Thus, although $C O P$ was formulated with reference to the eigenvalue method, it might be used as a quality test for any priority deriving methods, including the HRE approach.

\section{Heuristics of averaging with respect to reference values - the form of the linear equation system}

For simplicity, let us assume that $C_{U}=\left\{c_{1}, \ldots, c_{k}\right\}, C_{K}=\left\{c_{k+1}, \ldots, c_{n}\right\}$. The values $\mu$ for $c_{j} \in C_{K}$ are known, whilst the values $\mu$ for elements of $C_{U}$ need to be estimated. The heuristics of averaging with respect to the reference values assumes that for every unknown $c_{j} \in C_{U}$ the value $\mu\left(c_{j}\right)$ should be estimated as the arithmetic mean of all the other values $\mu\left(c_{i}\right)$ multiplied by factor $m_{j i}$ :

$$
\mu\left(c_{j}\right)=\frac{1}{n-1} \sum_{i=1, i \neq j}^{n} m_{j i} \mu\left(c_{i}\right)
$$

Thus, during the second and subsequent iterations the algorithm shown in [25] calculates the new estimation value $\mu\left(c_{i}\right)$ for each unknown concepts $c_{j} \in C_{U}$ according to one of the following equations:

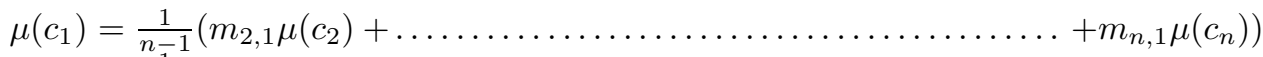

$$
\begin{aligned}
& \mu\left(c_{2}\right)=\frac{1}{n-1}\left(m_{1,2} \mu\left(c_{1}\right)+m_{3,2} \mu\left(c_{3}\right)+\ldots \ldots \ldots \ldots \ldots \ldots \ldots \ldots \ldots \ldots+m_{n, 2} \mu\left(c_{n}\right)\right) \\
& \mu\left(c_{k}\right)=\frac{1}{n-1}\left(m_{1, k} \mu\left(c_{1}\right)+\ldots+m_{k-1, k} \mu\left(c_{k-1}\right)+m_{k+1, k} \mu\left(c_{k+1}\right)+\ldots+m_{n, k} \mu\left(c_{n}\right)\right)
\end{aligned}
$$

Since the values $\mu\left(c_{k+1}\right), \ldots, \mu\left(c_{n}\right)$ are known and constant $\left(c_{k+1}, \ldots, c_{n}\right.$ are the reference concepts), so they can be grouped together. Let us denote:

$$
b_{j}=\frac{1}{n-1} m_{k+1, j} \mu\left(c_{k+1}\right)+\ldots+\frac{1}{n-1} m_{n, j} \mu\left(c_{n}\right)
$$

Thus, the linear equations system (60) could be written as:

$$
\begin{aligned}
& \mu\left(c_{1}\right)=\frac{1}{n-1} m_{2,1} \mu\left(c_{2}\right)+\ldots \ldots \ldots \ldots \ldots \ldots+\frac{1}{n-1} m_{k, 1} \mu\left(c_{k}\right)+b_{1} \\
& \mu\left(c_{2}\right)=\frac{1}{n-1} m_{1,2} \mu\left(c_{1}\right)+\frac{1}{n-1} m_{3,2} \mu\left(c_{3}\right)+\ldots+\frac{1}{n-1} m_{k, 2} \mu\left(c_{k}\right)+b_{2}
\end{aligned}
$$

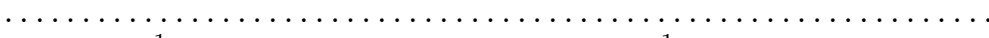

$$
\begin{aligned}
& \mu\left(c_{k}\right)=\frac{1}{n-1} m_{1, k} \mu\left(c_{1}\right)+\ldots \ldots \ldots \ldots \ldots+\frac{1}{n-1} m_{k-1, k} \mu\left(c_{k-1}\right)+b_{k}
\end{aligned}
$$

It is easy to see that the linear equation system (62) forms the matrix equation (8) where $A, b$ and $\mu$ are defined in (9), (10) and (11). Finding the solution of (8) is equivalent to determine the values $\mu\left(c_{1}\right), \ldots, \mu\left(c_{k}\right)$ with respect to the reference (known) concepts grouped in $C_{K}$. 